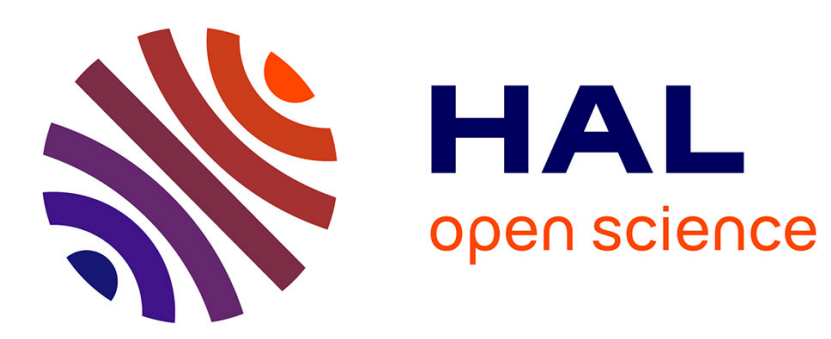

\title{
A Parametrized Ranking-based Semantics for Persuasion
}

Elise Bonzon, Jérôme Delobelle, Sébastien Konieczny, Nicolas Maudet

\section{To cite this version:}

Elise Bonzon, Jérôme Delobelle, Sébastien Konieczny, Nicolas Maudet. A Parametrized Rankingbased Semantics for Persuasion. 11th International Conference on Scalable Uncertainty Management (SUM 2017), Oct 2017, Grenade, Spain. pp.237-251, 10.1007/978-3-319-67582-4_17 . hal-01650122

\section{HAL Id: hal-01650122 \\ https://hal.science/hal-01650122}

Submitted on 28 Nov 2017

HAL is a multi-disciplinary open access archive for the deposit and dissemination of scientific research documents, whether they are published or not. The documents may come from teaching and research institutions in France or abroad, or from public or private research centers.
L'archive ouverte pluridisciplinaire HAL, est destinée au dépôt et à la diffusion de documents scientifiques de niveau recherche, publiés ou non, émanant des établissements d'enseignement et de recherche français ou étrangers, des laboratoires publics ou privés. 


\title{
A Parametrized Ranking-based Semantics for Persuasion
}

\author{
Elise Bonzon ${ }^{1}$, Jérôme Delobelle ${ }^{2}$, Sébastien Konieczny ${ }^{2}$, and Nicolas Maudet ${ }^{3}$ \\ ${ }^{1}$ LIPADE, Université Paris Descartes, France \\ ${ }^{2}$ CRIL, CNRS - Université d'Artois, France \\ ${ }^{3}$ Sorbonne Universités, UPMC Univ Paris 06, CNRS - LIP6, UMR 7606, France \\ bonzon@parisdescartes.fr \{delobelle, konieczny\}@cril.fr \\ nicolas.maudetelip6.fr
}

\begin{abstract}
In this paper we question the ability of the existant ranking semantics for argumentation to capture persuasion settings, emphasizing in particular the phenomena of protocatalepsis (the fact that it is often efficient to anticipate the counter-arguments of the audience), and of fading (the fact that long lines of argumentation become ineffective). It turns out that some widely accepted principles of ranking-based semantics are incompatible with a faithful treatment of these phenomena. We thus propose a parametrized semantics based on propagation of values, which allows to control the scope of arguments to be considered for evaluation. We investigate its properties (identifying in particular threshold values guaranteeing that some properties hold), and report experimental results showing that the family of rankings that may be returned have a high coherence rate.
\end{abstract}

Keywords: Argumentation, Persuasion, Ranking semantics

\section{Introduction}

Recently, the quest for a principled method to analyse networks of contradictory arguments has stimulated a number of work. Taken in their abstract form, such networks are argumentation frameworks, as defined by Dung [10]. Sharing the view that identifying sets of mutually acceptable arguments (extensions) is sometimes not sufficient, many "gradual" (returning a value) $[3,17,9,16]$ or "ranking" (returning an order) semantics have been proposed $[7,1,19,13,20,2,6]$. Each of these proposals has some merit, and nicely designed examples convince indeed that, in some situations at least, they should be the method of choice. When it comes to comparing these approaches (beyond their formal properties like convergence or uniqueness of solution), things become difficult. This is so because the basis of comparison is not so clear in the first place, different proposals emphasizing different properties. In [5], many existing semantics were compared on the basis on all the axioms mentioned in the literature. However, even the relevance of some axioms may be very much dependent on the context of application. What is often missing to compare these approaches is thus a clear indication of the applications they target.

In this paper, we aim at defining a good ranking semantics for persuasion. In this context, what constitutes an efficient argumentation has been rather extensively studied, 
and may constitute an interesting basis for comparaison. We shall concentrate on two well documented phenomena:

- Procatalepsis: anticipating the counter-arguments of an audience [22] is often a way to strengthen his own arguments, and many phenomena are well documented. To illustrate this, we extend an example from Besnard and Hunter[4][p.85]: a (made-up) sales pitch intended to persuade to buy a specific car.

(a1) The car $x$ is a high performance family car with a diesel engine and a price of 32000

(a2) In general, diesel engines have inferior performance compared with gazoline engines

(a3) But, with these new engines, the difference in performance [...] is negligible

(a4) In addition, even if the price of the car seems high

(a5) It will be amortized because the Diesel engines run longer before breaking than any kind of engines.

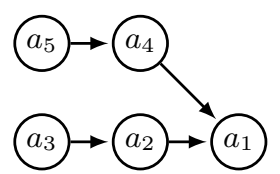

Apart from the fact that the example predates recent diesel scandals, what is striking is that it blatantly contradicts an axiom "Void Precedence" satisfied (to the best of our knowledge) by all gradual semantics and which considers non-attacked arguments as the most acceptable arguments. In this kind of persuasion contexts, it is clearly more convincing to state the more plausible counter-argument to $\left(a_{1}\right)$ in order to provide some convincing defenses against them, that simply state $\left(a_{1}\right)$ alone.

- Fading: Long lines of argumentation become ineffective in practice, because the audience easily looses track of the relation between the arguments. This is supported by recent evidence [21] which shows (in the context of their study, an extensive analysis of debates which took place on the subreddit "ChangeMyView"), that the arguments located at a distance of 10 from an other argument is about the limit. While some ranking semantics incorporate features which can be used to discount the strength of arguments relatively to their distance, this is not the case of all semantics.

We conclude that current ranking semantics are poorly equipped to be used in a context of persuasion. Our research question is thus to design a ranking-based semantics suited for persuasion, catering in particular for the fading effect and the fact void precedence might not be satisfied.

Our contribution is a gradual semantics which permits to account for these phenomena. Most importantly, some parameter allows to regulate how these principles are respected. Our vision is that, equipped with our ranking semantics, a seller facing different sales pitch might decide which is more likely to make the case. In general, this contribution could thus be used as an ingredient for developing strategies for computation persuasion techniques [14].

The remainder of this paper is as follows. In Section 2 we recall the necessary background in formal argumentation. Section 3.1 presents the basic principle of propagation 
on which our proposal, detailed in Section 3.2, is built. This semantics takes a parameter, the attenuation factor $\delta$, which allows to control the convergence speed and the obtained rankings. More importantly, we study in detail the relation between this factor and the "Void Precedence" axiom. In order to allow proper comparison with other proposals in the literature, the paper concludes with a study of the axiomatic properties of our semantics, as well as an extended example.

\section{Preliminaries}

Following Dung, we define an argumentation framework (AF) as a binary attack-relation over a (finite) set of abstracts arguments.

Definition 1 ([10]). An argumentation framework (AF) is a pair $F=\langle\mathcal{A}, \mathcal{R}\rangle$ where $\mathcal{A}$ is a set of arguments, and $\mathcal{R} \subseteq \mathcal{A} \times \mathcal{A}$ is a binary relation called the attack relation. Notation $(a, b) \in \mathcal{R}$ means that a attacks $b$. Let $\operatorname{Arg}(F)=\mathcal{A}$.

One of the main goals of argumentation theory is to identify which arguments are rationally acceptable according to different notions of acceptability. In [10], the acceptability of an argument depends on its membership to some extensions, whereas ranking-based semantics aim to rank arguments from the most to the least acceptable ones.

Definition 2. A ranking semantics $\sigma$ associates to any argumentation framework $F=$ $\langle\mathcal{A}, \mathcal{R}\rangle$ a ranking $\succeq_{F}^{\sigma}$ on $\mathcal{A}$, where $\succeq_{F}^{\sigma}$ is a preorder (a reflexive and transitive relation) on $\mathcal{A}$. $a \succeq_{F}^{\sigma} b$ means that $a$ is at least as acceptable as $b\left(a \simeq_{F}^{\sigma} b\right.$ is a shortcut for $a \succeq_{F}^{\sigma} b$ and $b \succeq_{F}^{\sigma} a$, and $a \succ_{F}^{\sigma} b$ is a shortcut for $a \succeq_{F}^{\sigma} b$ and $b \succeq_{F}^{\sigma} a$ ).

When the ranking semantics $\sigma$ and the graph $F$ is clear from the context, we will use $\succeq$ instead of $\succeq_{F}^{\sigma}$.

Let us introduce some notations that help us to define our ranking semantics in the next section.

Notation 1 Let $F=\langle\mathcal{A}, \mathcal{R}\rangle$ and $a, b \in \mathcal{A}$. A path from a to $b$, denoted by $p(a, b)$, is a sequence of nodes $s=\left\langle a_{0}, \ldots, a_{n}\right\rangle$ such that from each node there is an edge to the next node in the sequence: $a_{0}=a, a_{n}=b$ and $\forall i<n,\left(a_{i}, a_{i+1}\right) \in \mathcal{R}$. Its length is denoted by $|p(a, b)|$ and is equal to the number of edges it is composed of.

Notation 2 Let $\Delta_{n}(\mathrm{a})=\{b \mid \exists p(b, a)$, with $|p(b, a)|=n\}$ be the set of arguments that are bound by $a$ path of length $n$ to the argument $a$. An argument $b \in \Delta_{n}(a)$ is $a$ defender (resp. attacker) of a if $n$ is even (resp. odd). A path from b to a is a branch if $b$ is not attacked, i.e. if $\Delta_{1}(b)=\emptyset$. It is a defense branch (resp. attack branch) if $b$ is $a$ defender (resp. attacker) of $a . \Delta^{B^{+}}(a)$ (resp. $\left.\Delta^{B^{-}}(a)\right)$ denotes the set of all the defense (resp. attack) branches of $a$.

While our method is general, in the context of this paper we shall also pay special attention to tree shaped argumentation frameworks where an argument $a$, called root argument, has only defense branches (i.e. $\Delta^{B^{-}}(a)=\emptyset$ and $\Delta^{B^{+}}(a) \neq \emptyset$ ). Such frameworks will be called persuasion pitches. The AF in the introduction is an example of persuasion pitch with $a_{1}$ as root argument. 


\section{Variable-Depth Propagation}

\subsection{The propagation principle}

The semantics we propose in this paper follows the principle of propagation already used by some ranking semantics $[20,6]$. In short, the idea is to assign a positive initial value to each argument in the AF (arguments may start with the same initial value [20] or start with distinct values like in [6], where non-attacked arguments have greater value than attacked ones). Then each argument propagates its value into the argumentation framework, alternating the polarity according to the considered path (negatively if it is an attack path, positively if it is a defense one).

Inspired by these definitions, we formally define this propagation principle, including in addition a damping factor $\delta$ which allows to decrease the impact of attackers situated further away along a path (the longer the path length $i$, the smaller the $\delta^{i}$ ). Among other things, such a damping factor will allow to guarantee the convergence of the computation of the arguments' values, as also proposed in [18,20].

Definition 3. Let $\langle\mathcal{A}, \mathcal{R}\rangle$ be an argumentation framework. The valuation $P$ of a $\in \mathcal{A}$, at step $i$, is given by:

$$
P_{i}^{\epsilon, \delta}(a)= \begin{cases}v_{\epsilon}(a) & \text { if } i=0 \\ P_{i-1}^{\epsilon, \delta}(a)+(-1)^{i} \delta^{i} \sum_{b \in \Delta_{i}(a)} v_{\epsilon}(b) & \text { otherwise }\end{cases}
$$

with $\delta \in] 0,1\left[\right.$ be an attenuation factor and $v_{\epsilon}: \mathcal{A} \rightarrow \mathbb{R}^{+}$a valuation function that assigns an initial weight to each argument, with $\epsilon \in[0,1]$ such that $\forall b \in \mathcal{A}, v_{\epsilon}(b)=1$ if $\Delta_{1}(b)=\emptyset ; v_{\epsilon}(b)=\epsilon$ otherwise.

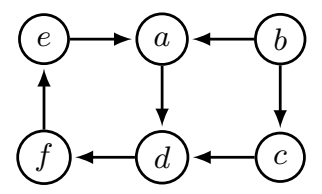

Fig. 1. The argumentation framework $F_{1}$

Example 1 Let us compute the valuation $P$ of each argument in $F_{1}$ (see Figure 1 ) when $\epsilon=0.5$ and $\delta=0.4$ and give the results in the Table 1 .

In focusing on the argument $f$, we can see that it begins with an initial weight of 0.5 $\left(P_{0}^{0.5,0.4}(f)=0.5\right)$ because it is attacked. Then, it receives negatively the value sent by its direct attacker $d$ which is also attacked: $P_{1}^{0.5,0.4}(f)=P_{0}^{0.5,0.4}(f)-0.4 \times v_{0.5}(d)=$ 0.3. Then, during the second step $(i=2)$, it receives positively the weights from a and c attenuated by $\delta^{2}: P_{2}^{0.5,0.4}(f)=P_{1}^{0.5,0.4}(f)+0.4^{2} \times\left(v_{0.5}(a)+v_{0.5}(c)\right)=0.46$. When $i=3$, it receives negatively the weight of 1 from $b$ and the weight of 0.5 from $e$ attenuated by $\delta^{3}: P_{3}^{0.5,0.4}(f)=P_{2}^{0.5,0.4}(f)-0.4^{3} \times\left(v_{0.5}(b)+v_{0.5}(e)\right)=0.364$. And so on and so forth. 


\begin{tabular}{|c|c|c|c|c|c|c|}
\hline$P_{i}^{0.5,0.4}$ & $a$ & $b$ & $c$ & $d$ & $e$ & $f$ \\
\hline 0 & 0.5 & 1 & 0.5 & 0.5 & 0.5 & 0.5 \\
1 & -0.1 & 1 & 0.1 & 0.1 & 0.3 & 0.3 \\
2 & -0.02 & 1 & 0.1 & 0.34 & 0.38 & 0.46 \\
3 & -0.052 & 1 & 0.1 & 0.308 & 0.316 & 0.364 \\
$\vdots$ & $\vdots$ & $\vdots$ & $\vdots$ & $\vdots$ & $\vdots$ & $\vdots$ \\
14 & -0.0402 & 1 & 0.1 & 0.3161 & 0.3506 & 0.3736 \\
\hline
\end{tabular}

Table 1. Computation of the valuation $\mathrm{P}$ of each argument from $F_{1}$ when $\epsilon=0.5$ and $\delta=0.4$

The following proposition answers to the question of convergence of the valuation $P$ for each argument for every AF.

Proposition 1 Let $\langle\mathcal{A}, \mathcal{R}\rangle$ be an argumentation framework and $\delta \in] 0,1[$. For all $a \in$ $\mathcal{A}$, the sequence $\left\{P_{i}^{\epsilon, \delta}(a)\right\}_{i=0}^{+\infty}$ converges.

The propagation number of an argument $a$ the value $P^{\epsilon, \delta}(a)=\lim _{i \rightarrow+\infty} P_{i}^{\epsilon, \delta}(a)$.

Example 1 (cont.) The propagation number of each argument from $F_{1}$ (see Figure 1) is represented in the shaded cell in the Table 1.

\subsection{The two-phase propagation method}

The non-attacked arguments play a key role for assessing the acceptability of arguments in Dung's classical semantics. Although, as explained before, we do not necessarily want them ranked above any other, these arguments must keep a specific role, at least to distinguish attack and defense branches (as also suggested in the global valuation approach of Cayrol and Lagasquie-Schiex [7]). Our solution is a two-phase process. In the first phase, non-attacked arguments propagate their weights $(=1)$ in the argumentation graph, while attacked arguments have weight 0 . Any pairwise strict comparison (based on propagation number) resulting from this process is fixed. In the second phase (that is, to break ties among arguments equally valued in the first phase), we re-run the propagation phase, this time setting an initial weight $\epsilon \neq 0$. Formally:

Definition 4. Let $\epsilon \in] 0,1]$ and $\delta \in] 0,1[$. The ranking-based semantics Variable-Depth Propagation $v d p^{\epsilon, \delta}$ associates to any argumentation framework $\langle\mathcal{A}, \mathcal{R}\rangle$ a ranking $\succeq$ on $\mathcal{A}$ such that $\forall a, b \in \mathcal{A}$ :

$$
a \succeq b \text { iff } P^{0, \delta}(a)>P^{0, \delta}(b) \text { or }\left(P^{0, \delta}(a)=P^{0, \delta}(b) \text { and } P^{\epsilon, \delta}(a) \geq P^{\epsilon, \delta}(b)\right)
$$

Example 1 (cont.) According to the previous definition, we need first to compute the propagation number of each argument with $\epsilon=0$. We obtain the following propagation numbers: $P^{0,0.4}(a)=-0.4105, P^{0,0.4}(b)=1, P^{0,0.4}(c)=-0.4, P^{0,0.4}(d)=$ $0.1642, P^{0,0.4}(e)=0.0263$ and $P^{0,0.4}(f)=-0.0656$. Thus, we can already obtain the following ranking:

$$
b \succ d \succ e \succ f \succ c \succ a
$$

Note that no arguments are equally acceptable here, so it is not necessary to perform the second phase. 
A concern might be that the value of $\epsilon$ used in the second phase might change the ranking obtained. We show that this is not the case:

Proposition 2 Let $F=\langle\mathcal{A}, \mathcal{R}\rangle$ be an argumentation framework and $\delta \in] 0,1\left[\forall \epsilon, \epsilon^{\prime} \in\right.$ ]0,1],

$$
v d p^{\epsilon, \delta}=v d p^{\epsilon^{\prime}, \delta}
$$

Please note that even if different values of $\epsilon$ do not change the preorder, it is necessary to keep it in the process in order to make a distinction between non-attacked and attacked arguments (see Definition 3 about the valuation function $v_{\epsilon}$ ). However, this is a purely internal artefact without any effect on the outcome of the method. To make this clear, we note $\operatorname{vdp}^{\delta}$ instead of $\operatorname{vdp}^{\epsilon, \delta}$ to describe our parametrized ranking semantics in general.

Now regarding $\delta$, two different values can produce different preorders. Indeed, this parameter allows to choose the scope of influence of the arguments in the system in addition to allow the convergence of the valuation $P$. For instance, with a value of $\delta$ close to 0 , only the nearest arguments (so a little part of the AF) are taken into consideration to compute the different propagation numbers whereas with a value of $\delta$ close to 1, (almost) all the AF will be inspected. Following the principle of the fading effect, it is natural to assume that arguments located at a long distance from another argument become ineffective. In terms of design, it seems very interesting to have the ability to control this parameter so as to specify a maximal depth after which arguments see their influence on the value of others vanish.

To better understand how to take this principle into account, let us inspect the algorithm used to compute the propagation numbers. First, a positive number is assigned to each argument $\left(\forall a \in \mathcal{A}, P_{0}^{\epsilon, \delta}(a)=1\right.$ if $a$ is non-attacked or $P_{0}^{\epsilon, \delta}(a)=\epsilon$ otherwise). Then at step $i \in \mathbb{N}$, we add (or remove) the accumulated score until the previous step $\left(P_{i-1}^{\epsilon, \delta}(a)\right)$ and the attenuated weights $\left(v_{\epsilon}\right.$ and $\left.\delta^{i}\right)$ received from arguments at the beginning of a path with a length of $i\left(\Delta_{i}\right): P_{i}^{\epsilon, \delta}(a)=P_{i-1}^{\epsilon, \delta}(a)+(-$ $1)^{i} \delta^{i} \sum_{b \in \Delta_{i}(a)} v_{\epsilon}(b)$. We stop the process when, between two steps, the difference with $b \in \Delta_{i}(a)$

the previous step for all the valuations $P$ is smaller than a fixed precision threshold $\mu$, i.e. $\forall a \in \mathcal{A},\left|P_{i}^{\epsilon, \delta}(a)-P_{i-1}^{\epsilon, \delta}(a)\right|<\mu$. Thus, given a precision, one can choose $\delta$ according to the maximal expected depth.

Proposition 3 Let $F$ be an argumentation framework, $i \in \mathbb{N} \backslash\{0\}$ be the maximal depth and $\mu$ be the precision threshold. If $\delta<\sqrt[i]{\frac{\max _{a \in \operatorname{Arg}(F)}\left(\left|\Delta_{i}(a)\right|\right)}{m^{2}}}$ then the sequence $\left\{P_{i}^{\epsilon, \delta}(a)\right\}_{i=0}^{+\infty}$ converges before step $i+1$.

Example 1 (cont.) Suppose that one considers that the maximal depth should be 5. In using the previous formula with a precision $\mu=0.0001$, then $\delta$ should be smaller than $\sqrt[5]{\frac{0.0001}{3}} \simeq 0.127$. Thus, a value close to this limit, for instance $\delta=0.12$, ensures that only the arguments until a depth of 5 (included) are considered.

We can also find a computational advantage to represent the fading effect. Indeed, as the number of steps needed to find the propagation number of each argument is smaller as if we need to browse all the AF, the ranking is computed faster. 


\section{Ranking-based properties}

We now investigate the properties satisfied by vdp. We start by inspecting the case of Void Precedence, before checking other properties discussed in the literature. These results give us some invariants, i.e. constraints on the resulting rankings. As we have seen, by tuning the parameter $\delta$, different rankings can still be returned, it is why we report experimental results showing that the diversity of rankings remains small.

\subsection{Void Precedence}

One of the very distinctive feature of vdp is that an attacked argument can have a better score (and so a better rank) than a non-attacked argument. Indeed, when a given argument has many defense branches and few attack branches, it receives many positive weights. Thus, according to the choice of $\delta$, this argument can obtain a greater score than the score of non-attacked arguments.

Void Precedence (VP) A non-attacked argument is ranked strictly higher than any attacked argument: $\Delta_{1}(a)=\emptyset$ and $\Delta_{1}(b) \neq \emptyset \Rightarrow a \succ b$

Let us illustrate that with the persuasion pitch used by the salesman in the introduction.

Example 2 Consider the AF illustrated in the introduction, where the argument $a_{1}$ has two defense branches. In computing the propagation number of each argument, with $\delta=0.95$ and $\epsilon=0$, we obtain $P^{0,0.95}\left(a_{1}\right)=1.805, P^{0,0.95}\left(a_{2}\right)=P^{0,0.95}\left(a_{4}\right)=$ -0.95 and $P^{0,0.95}\left(a_{3}\right)=P^{0,0.95}\left(a_{5}\right)=1$. With a non-zero $\epsilon=0.5, P^{0.5,0.95}\left(a_{1}\right)=$ 1.36, $P^{0.5,0.95}\left(a_{2}\right)=P^{0.5,0.95}\left(a_{4}\right)=-0.45$ and $P^{0.5,0.95}\left(a_{3}\right)=P^{0.5,0.95}\left(a_{5}\right)=1$. So, one can infer the following preorder where $a_{1}$ is better ranked than non-attacked arguments:

$$
a_{1} \succ a_{3} \simeq a_{5} \succ a_{2} \simeq a_{4}
$$

In fact, there exists a threshold for the parameter $\delta$ below which VP is satisfied.

Proposition 4 Let $\langle\mathcal{A}, \mathcal{R}\rangle$ be an argumentation framework and $\delta^{M}=\sqrt{\frac{1}{\max _{a \in \mathcal{A}}\left(\left|\Delta_{2}(a)\right|\right)}}$

$$
\text { If } \delta<\delta^{M} \text { then } v d p^{\delta} \text { satisfies } V P
$$

Let us check which values of $\delta$ are needed to satisfy VP for the argumentation framework in the introduction:

Example 2 (cont.) The argument $a_{1}$ has the highest number of direct defenders with $\left|\Delta_{2}\left(a_{1}\right)\right|=2$. The value of $\delta$ should be now $: \delta<\delta^{M}=\sqrt{1 / 2} \simeq 0.7071$. So if $\delta=0.7$, we obtain $P^{0,0.7}\left(a_{1}\right)=0.98, P^{0,0.7}\left(a_{2}\right)=P^{0,0.7}\left(a_{4}\right)=-0.7$ and $P^{0,0.7}\left(a_{3}\right)=$ $P^{0,0.7}\left(a_{5}\right)=1$ when $\epsilon=0$ and $P^{0.5,0.7}\left(a_{1}\right)=0.78, P^{0.5,0.7}\left(a_{2}\right)=P^{0.5,0.7}\left(a_{4}\right)=$ -0.2 and $P^{0.5,0.7}\left(a_{3}\right)=P^{0.5,0.7}\left(a_{5}\right)=1$ when $\epsilon=0.5$. These results allow to obtain the following preorder: $a_{3} \simeq a_{5} \succ a_{1} \succ a_{2} \simeq a_{4}$. 
The question to know if VP should hold or not relates to the status of the missing information in argumentation systems. If all the information are available, then "really unattacked" arguments should be better that any attacked argument, as it is the case with the other semantics. But there are cases where the argumentation systems encode the information currently available, and that is susceptible to be completed. This is this case that we attack in this paper with procatalepsis. Non-attacked argument can be seen as an argument which has not yet been debated whereas it is more difficult to find counter-arguments to an argument already attacked but defended thereafter.

Thus, our method departs from other approaches in its treatment of the VP property, but to a certain extent only. For instance, in a persuasion pitch a single line of defense is not enough to be more convincing than a non-attacked argument. On the other hand, when this condition is met a simple condition for the violation of VP in persuasion pitches can be stated:

Proposition 5 Let $P P=\langle\mathcal{A}, \mathcal{R}\rangle$ be a persuasion pitch with a $\in \mathcal{A}$ as the root argument. Then,

(i) if $\left|\Delta^{B^{+}}(a)\right|<2$ then $v d p^{\delta}$ satisfies $V P$;

(ii) if $\left|\Delta^{B^{+}}(a)\right| \geq 2$ and $\delta>\sqrt[m]{\frac{1}{\mid \Delta^{B^{+}(a) \mid}}}$ with $m$ the length of the longest defense branch of a then $v d p^{\delta}$ violates $V P$.

Let us discuss about the link between the two principles concerning $\delta$. Indeed, the value of $\delta$ should not be too small in order to take into account enough arguments (and not only the direct attackers for example) to obtain a significant result. But, in the same way, it also should not be too high if one wants to capture the procatalepsis principle. Understanding this interplay can provide valuable information, in particular in the persuasion context. Suppose for instance that the persuader knows that a given $\delta$ value is expected, corresponding to the profile of a specific audience. Then, this value being fixed, it is possible to infer that a certain number of defense branches will be required. Hence, instead of developing, say, two long lines of persuasion, the persuader will instead favor the deployment of a number of alternative lines in her persuasion pitch.

Interestingly, it turns out that in the context of our method, the property VP is related with another property studied in the literature, namely defense precedence:

Defense Precedence (DP) For two arguments with the same number of direct attackers, a defended argument is ranked higher than a non-defended argument:

$$
\left|\Delta_{1}(a)\right|=\left|\Delta_{1}(b)\right|, \Delta_{2}(a) \neq \emptyset \text { and } \Delta_{2}(b)=\emptyset \Rightarrow a \succ b
$$

Proposition 6 If $v d p^{\delta}$ satisfies VP then it satisfies DP.

Note that this is not the case in general (some semantics satisfy VP but not DP).

\subsection{Other properties}

Several other properties have been proposed, and studied in the literature (see [5] for an overview). Below we study how our method stands with respect to these properties. 
We give their informal definition and point the reader to [5] for the complete versions. Basic general properties are the fact that a ranking on a set of arguments should only depend on the attack relation (Abstraction, Abs); that the ranking between two arguments should be independent of arguments that are not connected to either of them (Independence, In); that all arguments can be compared (Total, Tot); and that all non-attacked arguments should be equally acceptable (Non-attacked Equivalence, NaE).

Local properties (like the already introduced DP) confine themselves to the level of direct attackers: (Counter-Transitivity, CT) states that if the direct attackers of $b$ are (i) at least as numerous and (ii) acceptable as those of $a$, then $a$ should be at least as acceptable as $b$, while in its strict version (SCT) either (i) or (ii) must be strict, implying a strict comparison between $a$ and $b$.

Global properties specify how the ranking should be affected on the basis of the comparison of attack and defense branches. More precisely: adding a defense branch to an attacked argument should increase its acceptability (Addition of Defense Branch, +DB); increasing the length of an attack branch of an argument should increase its acceptability (Increase of Attack Branch, $\uparrow \mathbf{A B}$ ); adding an attack branch to an argument should decrease its acceptability (Addition of Attack Branch, $+\mathbf{A B}$ ); and increasing the length of a defense branch of an argument should decrease its acceptability (Increase of Defense Branch, $\uparrow \mathbf{D B})$. Note that $+\mathrm{DB}$ is indeed restricted to attacked arguments, otherwise its incompatibility with VP is obvious. In the same spirit, (Attack vs Full Defense, AvsFD), i.e. the fact that an argument with only defense branches and no attack branch should be strictly more acceptable than an argument attacked once by a non-attacked argument. For persuasion pitches, this property can be simply reformulated as "a persuasion pitch for $x$ should make it more acceptable than stating $x$ with an attacking argument". This seems compelling in our context, thus providing further evidence of the inability of many of the existing semantics to properly capture persuasion settings.

Let us now check which properties are satisfied by vdp :

Proposition 7 Let $\delta \in] 0,1[$. vdp $\delta$ satisfies Abs, In, Tot, NaE, $+A B$ and AvsFD.

Some global properties like $+\mathrm{DB}, \uparrow \mathrm{DB}$ and $\uparrow \mathrm{AB}$ are not satisfied because of the fading effect. Indeed, when the branch, which is added or extended, is too long, the arguments at the end of this branch have no impact on the targeted argument. It is why, we propose to define the corresponding properties $\left(+\mathrm{DB}_{i}, \uparrow \mathrm{DB}_{i}\right.$ and $\left.\uparrow \mathrm{AB}_{i}\right)$ which capture the same idea but with the additional condition that the maximal length of the branch is $i$.

Proposition 8 With $\delta \in] \delta^{m}, 1\left[\right.$ s.t. $\delta^{m}=\sqrt[i]{\frac{\mu}{\max _{a \in \operatorname{Arg}(F)}\left(\left|\Delta_{i}(a)\right|\right)}}$ where $i$ represents the length of the branch which is added or extended then $v d p^{\delta}$ satisfies also $+D B_{i}, \uparrow D B_{i}$ and $\uparrow A B_{i}$.

These results are reported in Table 2. For comparison purpose, we also include in this table the results of some semantics from the literature where the same set [5] of properties has been already checked. Namely, these semantics are: the semantics based on Social Argumentation Frameworks $S A F[16,11,8]$ restricted to Dung's argumentation framework, the semantics Categoriser Cat [3,19], the Discussion-based semantics 
$D b s$ and the Burden-based semantics $B b s$ [1], the global semantics based on tuple values Tuples* [7], and the semantics $M \& T$ [17].

\begin{tabular}{|c||c|c|c|c|c|c||c|c|}
\hline Properties & SAF & Cat & Dbs & Bbs & Tuples $^{*}$ & M\&T & vdp $^{\delta}$ & vdp $^{\delta^{\prime}}$ \\
\hline \hline Abs & $\checkmark$ & $\checkmark$ & $\checkmark$ & $\checkmark$ & $\checkmark$ & $\checkmark$ & $\checkmark$ & $\checkmark$ \\
\hline In & $\checkmark$ & $\checkmark$ & $\checkmark$ & $\checkmark$ & $\checkmark$ & $\checkmark$ & $\checkmark$ & $\checkmark$ \\
\hline Tot & $\checkmark$ & $\checkmark$ & $\checkmark$ & $\checkmark$ & $\times$ & $\checkmark$ & $\checkmark$ & $\checkmark$ \\
\hline NaE & $\checkmark$ & $\checkmark$ & $\checkmark$ & $\checkmark$ & $\checkmark$ & $\checkmark$ & $\checkmark$ & $\checkmark$ \\
\hline+ AB & $\checkmark$ & $\checkmark$ & $\checkmark$ & $\checkmark$ & $\checkmark$ & $\checkmark$ & $\checkmark$ & $\checkmark$ \\
\hline AvsFD & $\times$ & $\times$ & $\times$ & $\times$ & $\checkmark$ & $\checkmark$ & $\checkmark$ & $\checkmark$ \\
\hline+ DB & $\times$ & $\times$ & $\times$ & $\times$ & $\checkmark$ & $\times$ & $\times$ & $\checkmark i$ \\
\hline$\uparrow$ AB & $\checkmark$ & $\checkmark$ & $\checkmark$ & $\checkmark$ & $\checkmark$ & $\times$ & $\times$ & $\checkmark i$ \\
\hline$\uparrow$ DB & $\checkmark$ & $\checkmark$ & $\checkmark$ & $\checkmark$ & $\checkmark$ & $\times$ & $\times$ & $\checkmark i$ \\
\hline VP & $\checkmark$ & $\checkmark$ & $\checkmark$ & $\checkmark$ & $\checkmark$ & $\checkmark$ & $\times$ & $\checkmark$ \\
\hline DP & $\checkmark$ & $\checkmark$ & $\checkmark$ & $\checkmark$ & $\times$ & $\times$ & $\times$ & $\checkmark$ \\
\hline CT / SCT & $\checkmark$ & $\checkmark$ & $\checkmark$ & $\checkmark$ & $\times$ & $\times$ & $\times$ & $\times$ \\
\hline
\end{tabular}

Table 2. Summary of the properties satisfied by $\operatorname{vdp}\left(\forall \delta\right.$, and with $\left.\delta^{m}<\delta^{\prime}<\delta^{M}\right)$ and some existing ranking semantics from the literature where the same set of properties has been already checked. A cross $\times$ means that the property is not satisfied, symbol $\checkmark$ means that the property is satisfied and $\checkmark_{i}$ means that the $i$-version of the property (cf Property 8 ) is satisfied. Shaded cells are results proved in this paper.

We first remark that vdp satisfy the "basic" properties according to [5] (Abs, In, $+\mathrm{AB}, \mathrm{NaE}$ and Tot), at the exception of VP as intended by design and discussed earlier.

We can also note that vdp always satisfies property AvsFD, and for a specific $\delta$ the property + DB. Indeed, the possibility to rank arguments with various different defenders higher than arguments which are defended only once seems very interesting. For instance, in persuasion scenarios, a claim defended with various different arguments may be more credible than a claim only defended once.

In the end, the only properties which are never satisfied are CT and SCT. In fact, it is easy to show that these properties are incompatible with $+\mathrm{DB}$. Intuitively, whereas $+\mathrm{DB}$ considers that adding a defense is positive for an argument, SCT says that adding any branch (so including defense branch) to an argument should decrease its acceptability.

\subsection{On the diversity of rankings}

A nice feature of our semantics is thus that the designer can choose whether VP holds or not, giving rise to different rankings. However, one may be worried that the diversity of rankings is so high that the semantics becomes too sensitive to small modifications of the parameter $\delta$. To check this, we applied our semantics on 1000 randomly generated 
$\mathrm{AFs}^{1}$ for different values of $\delta \in\{0.001,0.2,0.4,0.6,0.8,0.9\}$. Then, we computed the similarity degree between two rankings from two different values of $\delta$ in using the Kendall tau distance [15] which returns a value between 0 and 1.

Definition 5. Let $\langle\mathcal{A}, \mathcal{R}\rangle$ and $\tau_{\sigma_{1}}, \tau_{\sigma_{2}}$ the orders returned by the ranking semantics $\sigma_{1}$ and $\sigma_{2}$ respectively. The Kendall tau distance between $\tau_{\sigma_{1}}$ and $\tau_{\sigma_{2}}$ is calculated as follow:

$$
K\left(\tau_{\sigma_{1}}, \tau_{\sigma_{2}}\right)=\frac{\sum_{\{i, j\} \in \mathcal{A}} \overline{K_{i, j}}\left(\tau_{\sigma_{1}}, \tau_{\sigma_{2}}\right)}{0.5 \times|\mathcal{A}| \times(|\mathcal{A}|-1)}
$$

with :

- $\overline{K_{i, j}}\left(\tau_{\sigma_{1}}, \tau_{\sigma_{2}}\right)=1$ if $i \succ^{\sigma_{1}} j$ and $i \succ^{\sigma_{2}} j$, or $i \prec^{\sigma_{1}} j$ and $i \prec^{\sigma_{2}} j$, or $i \simeq^{\sigma_{1}} j$ and $i \simeq \sigma_{2} j$,

- $\overline{K_{i, j}}\left(\tau_{\sigma_{1}}, \tau_{\sigma_{2}}\right)=0$ if $i \succ^{\sigma_{1}} j$ and $i \prec^{\sigma_{2}} j$ or vice versa,

- $\overline{K_{i, j}}\left(\tau_{\sigma_{1}}, \tau_{\sigma_{2}}\right)=0.5$ if $i \succ^{\sigma_{1}} j$ or $i \prec^{\sigma_{1}} j$ and $i \simeq^{\sigma_{2}} j$ or vice versa.

Thus, two rankings with a Kendall tau distance of 1 are fully similar whereas a score of 0 means that they are totally reversed. The Table 3 contains, for each pair of semantics, the average Kendall tau distance $(\times 100)$ computed on the 1000 generated AFs. The results show that the obtained rankings stay pretty close because the smallest observed similarity between the smallest and largest value of $\delta$ is $86.26 \%$. This similarity remains overall very high, showing that the semantics remains quite stable as the parameter varies.

\begin{tabular}{|c|c|c|c|c|c|c|}
\hline$\delta$ & $\mathbf{0 . 0 0 1}$ & $\mathbf{0 . 2}$ & $\mathbf{0 . 4}$ & $\mathbf{0 . 6}$ & $\mathbf{0 . 8}$ & $\mathbf{0 . 9}$ \\
\hline $\mathbf{0 . 0 0 1}$ & 100 & 99.94 & 99.45 & 95.91 & 89.38 & 86.26 \\
\hline $\mathbf{0 . 2}$ & 99.94 & 100 & 99.48 & 95.87 & 89.37 & 86.36 \\
\hline $\mathbf{0 . 4}$ & 99.45 & 99.48 & 100 & 96.29 & 89.87 & 86.70 \\
\hline $\mathbf{0 . 6}$ & 95.91 & 95.87 & 96.29 & 100 & 93.18 & 90.14 \\
\hline $\mathbf{0 . 8}$ & 89.38 & 89.37 & 89.87 & 93.18 & 100 & 96.84 \\
\hline $\mathbf{0 . 9}$ & 86.26 & 86.36 & 86.70 & 90.14 & 96.84 & 100 \\
\hline
\end{tabular}

Table 3. Average Kendall tau distance on 1000 randomly generated AFs for different values of $\delta$

\section{Comparison with Related Work}

Now, let us show that, in general, the different semantics proposed in the literature may return a large variety of rankings. To show this, we will use the example of Figure 1.

The range of semantics considered here is more important than the previous section because we include recent semantics for which the axiomatic properties have not

\footnotetext{
${ }^{1}$ The generation algorithms are based on the three algorithms used for producing the benchmarks of the competition ICCMA'15 (see http://argumentationcompetition. org/2015/results.html)
} 
(to the best of our knowledge) been yet studied. Two kinds of existing semantics are excluded from this study, because of specificities which make the comparison difficult. The first ones are the semantics which return a partial preorder between arguments (i.e. some arguments could be incomparable) like the global semantics based on tuple values [7] and the semantics proposed in [13]. The second category is the semantics that return a set of rankings for a same AF, like [12]. Thus, we consider the semantics Cat, $M \& T, S A F, D b s$ and Bbs that have already been mentioned on Section 4.2. We will also consider the semantics using the fuzzy label $F L$ [9], the $\alpha$-Burden-based semantics $\alpha$-BBS [2], the counting semantics $C S$ [20] and the propagation semantics $\operatorname{Propa}_{\epsilon}, \operatorname{Propa}_{1+\epsilon}, \operatorname{Propa}_{1 \rightarrow \epsilon}$ from [6]. All these rankings are represented in Fig. 2.

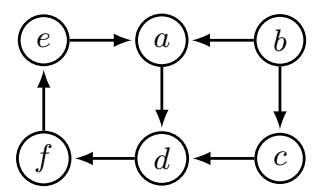

\begin{tabular}{|c|c|}
\hline Semantics & Order between arguments \\
\hline $\mathrm{M} \& \mathrm{~T}$ & $b \succ d \simeq e \succ a \simeq c \simeq f$ \\
\hline FL & $b \succ d \succ e \succ f \succ a \simeq c$ \\
\hline SAF & \multirow{4}{*}{$b \succ d \succ e \succ f \succ c \succ a$} \\
\hline$\alpha$-BBS $(\alpha=5)$ & \\
\hline Propa $_{1 \rightarrow \epsilon}$ & \\
\hline $\operatorname{vdp}^{\delta}(\delta=0.3)$ & \\
\hline $\mathrm{Dbs} / \mathrm{Bbs}$ & \multirow{4}{*}{$b \succ f \succ e \succ c \succ d \succ a$} \\
\hline $\mathrm{CS}$ & \\
\hline$\alpha-\operatorname{BBS}(\alpha=0.5)$ & \\
\hline $\operatorname{Propa}_{\epsilon}(\epsilon=0.8)$ & \\
\hline Cat & \multirow{3}{*}{$b \succ f \succ e \succ d \succ c \succ a$} \\
\hline $\operatorname{Propa}_{\epsilon}(\epsilon=0.3)$ & \\
\hline Propa $_{1+\epsilon}$ & \\
\hline $\operatorname{vdp}^{\delta}(\delta=0.8)$ & $d \succ b \succ e \succ c \succ f \succ a$ \\
\hline
\end{tabular}

Fig. 2. Orders obtained with the different ranking semantics applied to $F_{1}$

The particularity of our ranking semantics is clearly visible in this table because only vdp with $\delta \geq \frac{\sqrt{2}}{2}$ consider $d$ as strictly more acceptable than $b$. All the other semantics satisfy VP, so consider $b$, which is non-attacked, as the best argument in this AF. Indeed, thanks to the even cycle, $d$ receives only positive weights from the only nonattacked argument $b$ and is also defended by $e$. However, $d$ is not always among the best arguments as it is the case with the semantics which consider that a new defense branch can reinforce an argument (i.e. the semantics that satisfy +DB and AvsFD). Indeed, the semantics which consider a defense as a weak attack (i.e. the semantics that satisfy $\mathrm{SCT}$ ) judge that even if $d$ is defended, it is still directly attacked once more than $c, e$ and $f$. The reverse reasoning hold with the argument $f$ which is one of the best arguments for the semantics that satisfy SCT whereas is stay quite acceptable for the semantics that satisfy $+\mathrm{DB}$ and AvsFD. The worst arguments is always $a$ which is attacked twice including once by $b$ which is non-attacked. It is why it is almost always worst than $c$ which is directly attacked by $b$ only.

Finally, it is important to note that even if our proposal is related to $\mathrm{Pu}$ et al. [20] and 
Bonzon et al. [6] concerning the propagation method, it is substantially different, and has clearly different properties and behaviors (see Fig. 2).

\section{Conclusion}

Many ranking-based semantics have been proposed recently in the literature. Despite detailed studies of their properties, it remains hard to see which is more appropriate for a given application context. In this paper, we took the problem the other way and challenged these semantics in the context of persuasion, emphasizing in particular two well-documented phenomena occurring in practice: protocatalepsis and fading. It turns out that none of the proposed semantics is really appropriate - all of them commit for instance to the "Void Precedence" property which is incompatible with the procatalepsis principle. This motivated us to introduce a new parametrized ranking semantics based on the notion of propagation. An attenuation factor is used to allow the convergence but also to decrease the impact of further arguments. We show that, thanks to this attenuation factor, fading can be captured by selecting a maximal influence depth. For some values of this parameter VP is not satisfied, which allows to represent protocatalepsis in persuasion pitches. We also study other properties of our method, and we experimentally study how diverse can the rankings be depending of the value of the parameter. Future work include testing this semantics on currently developed computation persuasion tools.

Our methodology may also prove inspiring in other settings: by questioning the relevance of the existing semantics in other application contexts (e.g. negotiation), we may find out that some specific phenomena are not properly captured, and that other adjustments are required.

\section{Acknowledgements}

This work benefited from the support of the project AMANDE ANR-13-BS02-0004 of the French National Research Agency (ANR).

\section{References}

1. Leila Amgoud and Jonathan Ben-Naim. Ranking-based semantics for argumentation frameworks. In Proc. of the 7th International Conference on Scalable Uncertainty Management, (SUM'13), pages 134-147, 2013.

2. Leila Amgoud, Jonathan Ben-Naim, Dragan Doder, and Srdjan Vesic. Ranking arguments with compensation-based semantics. In Proc. of the 15th International Conference on Principles of Knowledge Representation and Reasoning, (KR'16), pages 12-21, 2016.

3. Philippe Besnard and Anthony Hunter. A logic-based theory of deductive arguments. Artificial Intelligence, 128(1-2):203-235, 2001.

4. Philippe Besnard and Anthony Hunter. Elements of Argumentation. MIT Press, 2008.

5. Elise Bonzon, Jérôme Delobelle, Sébastien Konieczny, and Nicolas Maudet. A Comparative Study of Ranking-based Semantics for Abstract Argumentation. In Proc. of the 30th AAAI Conference on Artificial Intelligence (AAAI'16), pages 914-920, 2016. 
6. Elise Bonzon, Jérôme Delobelle, Sébastien Konieczny, and Nicolas Maudet. Argumentation Ranking Semantics based on Propagation. In Proc. of the 6th International Conference on Computational Models of Argument (COMMA'16), pages 139-150, 2016.

7. Claudette Cayrol and Marie-Christine Lagasquie-Schiex. Graduality in argumentation. Journal of Artificial Intelligence Research, 23:245-297, 2005.

8. Marco Correia, Jorge Cruz, and João Leite. On the efficient implementation of social abstract argumentation. In Proc. of the 21st European Conference on Artificial Intelligence, (ECAI'14), pages 225-230, 2014.

9. Célia da Costa Pereira, Andrea Tettamanzi, and Serena Villata. Changing one's mind: Erase or rewind? In Proc. of the 22nd International Joint Conference on Artificial Intelligence, (IJCAI'11), pages 164-171, 2011.

10. Phan Minh Dung. On the acceptability of arguments and its fundamental role in nonmonotonic reasoning, logic programming and n-person games. Artificial Intelligence, 77(2):321358, 1995.

11. Sinan Egilmez Egilmez, João Martins, and João Leite. Extending social abstract argumentation with votes on attacks. In Proc. of the 2nd International Workshop on Theory and Applications of Formal Argumentation, (TAFA'13), pages 16-31, 2013.

12. Dov M. Gabbay. Equational approach to argumentation networks. Argument \& Computation, 3(2-3):87-142, 2012.

13. Davide Grossi and Sanjay Modgil. On the graded acceptability of arguments. In Proc. of the 24th International Joint Conference on Artificial Intelligence, (IJCAI'15), pages 868-874, 2015.

14. Anthony Hunter. Opportunities for argument-centric persuasion in behaviour change. In Proc. of the 14th European Conference on Logics in Artificial Intelligence, (JELIA'14), pages 48-61, 2014.

15. M. G. Kendall. A New Measure of Rank Correlation. Biometrika, 30(1/2):81-93, June 1938.

16. João Leite and João Martins. Social abstract argumentation. In Proc. of the 22nd International Joint Conference on Artificial Intelligence, (IJCAI'11), pages 2287-2292, 2011.

17. Paul-Amaury Matt and Francesca Toni. A game-theoretic measure of argument strength for abstract argumentation. In Proc. of the 11th European Conference on Logics in Artificial Intelligence, (JELIA'08), pages 285-297, 2008.

18. Fuan Pu, Jian Luo, and Guiming Luo. Some supplementaries to the counting semantics for abstract argumentation. In Proc. of the 27th IEEE International Conference on Tools with Artificial Intelligence (ICTAI'15), pages 242-249, 2015.

19. Fuan $\mathrm{Pu}$, Jian Luo, Yulai Zhang, and Guiming Luo. Argument ranking with categoriser function. In Proc. of the 7th International Conference on Knowledge Science, Engineering and Management, (KSEM'14), pages 290-301, 2014.

20. Fuan Pu, Jian Luo, Yulai Zhang, and Guiming Luo. Attacker and defender counting approach for abstract argumentation. In Proc. of the 37th Annual Meeting of the Cognitive Science Society, (CogSci'15), 2015.

21. Chenhao Tan, Vlad Niculae, Cristian Danescu-Niculescu-Mizil, and Lillian Lee. Winning arguments: Interaction dynamics and persuasion strategies in good-faith online discussions. In Proc. of the 25th International Conference on World Wide Web, (WWW'16), pages 613624, 2016.

22. Douglas Walton. Dialog Theory for Critical Argumentation. John Benjamins Publishing, 2007. 